\title{
Distributed Adaptive Synchronization for Complex Dynamical Networks with Uncertain Nonlinear Neutral-Type Coupling
}

\author{
Shi Miao and Li Junmin \\ Department of Mathematics, Xidian University, Xian 710071, China \\ Correspondence should be addressed to Li Junmin; jmli@mail.xidian.edu.cn
}

Received 10 July 2013; Revised 23 August 2013; Accepted 28 August 2013

Academic Editor: Zhiwei Gao

Copyright ( 92013 S. Miao and L. Junmin. This is an open access article distributed under the Creative Commons Attribution License, which permits unrestricted use, distribution, and reproduction in any medium, provided the original work is properly cited.

Distributed adaptive synchronization control for complex dynamical networks with nonlinear derivative coupling is proposed. The distributed adaptive strategies are constituted by directed connections among nodes. By means of the parameters separation, the nonlinear functions can be transformed into the linearly form. Then effective distributed adaptive techniques are designed to eliminate the effect of time-varying parameters and made the considered network synchronize a given trajectory in the sense of square error norm. Furthermore, the coupling matrix is not assumed to be symmetric or irreducible. An example shows the applicability and feasibility of the approach.

\section{Introduction}

A complex network is a large set of interconnected nodes, where the nodes represent individuals in the graph and the edges represent the connections among them, such as climate system [1], biological neural networks [2], human brain system [3]. Many natural and man-made systems can be modeled and characterized by complex networks successfully [4]. Such systems may be characterized by a system with uncertainties, time delays, nonlinearity, neutral properties, hybrid dynamics, distributed dynamics and chaotic dynamics.

Synchronization phenomena has been found in different forms in complex networks, such as fireflies in the forest, description of hearts, and routing messages in the internet. Thus synchronization is one of meaningful issues in dynamical characteristics of the complex dynamical networks. A considerable number of papers on this topic have appeared (see [5-7] and references therein.)

Recently, various control techniques have been reported to achieve networks synchronization (see $[4,8-16]$ and references therein) Some control schemes [8-13] were based on a solution of the homogenous system, in which it may be difficult to obtain the state information of an isolated node.
Consequently, utilizing the information from neighborhood to realize the network synchronization is more reasonable. Paper [17] introduced the concept of control topology to describe the whole controller structure. In [18], based on local information of node dynamics, an effective distributed adaptive strategy was designed to tune the coupling weights of a network. A considerable number of controlled synchronization techniques have been derived for complex dynamic networks based on the assumption which is the coupled nodes of CDN with the same dynamics (see the above papers and the references therein). In reality, complex networks are more likely to have different nodes for different dynamics. For example, in a multi-robot system, the robots can have distinct dynamic structures or different parameters. Recently, special attention has been focused on the synchronization of complex dynamical networks with nonidentical nodes [1922]. Paper [20] investigated the synchronization problem of a complex network with nonidentical nodes via openloop controllers. The paper [22] considered nonlinearly coupled networks with non-identical nodes and designed pinning control to obtain synchronization criteria. On the other hand, many real-world network systems' structure will change over time and contain unknown parameters. Very recently, some papers studied complex networks with 
unknown time-varying coupling strength [23-26]. In these results, non-identical nodes were not considered. Only in [21], the time-varying complex network with non-identical nodes was investigated, and a criterion of global bounded synchronization of the maximum state deviation between nodes was developed.

In other aspects, new complex networks models are proposed to reflect the complexity from the network structure. Thus, the problem of neutral-type couplings has also been widely investigated [27-31]. However, in the above studies, only linear derivative coupling is considered. More recently, [32] studied the synchronization in a class of dynamical networks with distributed delays and nonlinear derivative coupling. Considering the preceding discussion, nonidentical nodes complex dynamic network with nonlinear derivative coupling, and time-varying coupling strength is not concerned yet.

Inspired by the aforementioned results, the problem of adaptive synchronization is studied for complex dynamical networks with non-identical nodes, nonlinearly derivative couplings, and unknown time-varying coupling strength. A prominent feature of this network is that its complexity originates not only from the nonlinear dynamics of the nodes, but also from the complex coupling strength. The difficulty in dealing with the nonlinearly derivative couplings with unknown time-varying parameters is solved by using the parameter separation method. The distributed adaptive learning laws of periodically time-varying and constant parameters and the distributed adaptive controllers are constructed to guarantee that the system is asymptotically stable and that all closed loop signals are bounded.

The remainder of the paper is organized as follows. The problem statement and preliminaries are given in Section 2. Section 3 gives the main results and proofs. In Section 4, an illustrative example is provided to verify the theoretical results. Finally, conclusion is given.

\section{Problem Statement and Preliminaries}

The complex dynamical network is described as

$$
\begin{aligned}
\dot{x}_{i}(t)= & f_{i}\left(x_{i}(t)\right)+\sum_{J=1}^{N} a_{i j} \Gamma g\left(x_{j}(t), \varphi_{i}(t)\right) \\
& +\sum_{j=1}^{N} b_{i j} \Gamma k\left(\dot{x}_{j}(t), \beta_{i}(t)\right), \quad i=1,2, \ldots, N,
\end{aligned}
$$

where $x_{i}(t)=\left[x_{i 1}(t), x_{i 2}(t), \ldots, x_{i n}(t)\right]^{T} \in R^{n}$ is the state vector of the $i$ th node, $f_{i}(\cdot): R^{n} \rightarrow R^{n}$ is a vector-valued continuous function, and $g(\cdot, \cdot), k(\cdot, \cdot): R^{n} \times R \rightarrow R^{n}$ are unknown continuous nonlinear vector-valued functions, $\varphi_{i}(t), \beta_{i}(t)$ represent the unknown time-varying functions, the inner-coupling matrix $\Gamma=\operatorname{diag}\left(\gamma_{1}, \ldots, \gamma_{n}\right)$ is positive definite. $A=\left(a_{i j}\right)_{N \times N}$ and $B=\left(b_{i j}\right)_{N \times N}$ are the coupling configuration matrices, which satisfying $\sum_{j=1}^{N} a_{i j}=\sum_{j=1}^{N} b_{i j}=$ 0 and defined as follows: if there is a connection between node $i$ and node $j(i \neq j)$, then $a_{i j}>0, b_{i j}>0$, otherwise, $a_{i j}=b_{i j}=0$.
Remark 1. It should be pointed out that the nonlinear derivative couplings consist of more information in CDN than the linear derivative couplings in [28], the challenge problem in (1) is due to the uncertain nonlinear neutral-type coupling $\sum_{j=1}^{N} b_{i j} \Gamma k\left(\dot{x}_{j}(t), \beta_{i}(t)\right)$. For the unknown parameter $\beta_{i}(t)$ and the nonlinear function $k\left(\dot{x}_{j}(t), \beta_{i}(t)\right)$, in this paper, we shall overcome the obstacle though the domination technique and the parameters separation principle. Under later assumption, we can "separate" the parameters from the nonlinear function.

Define synchronization error as

$$
e_{i}(t)=x_{i}(t)-s(t), \quad i=1,2, \ldots, N
$$

where $s(t) \in R^{n}$ is a solution of the dynamics of the isolated node to which all $x_{i}(t)$ are expected to synchronize.

Then, (1) is said to be asymptotic synchronization in the $L_{T}^{2}$ norm sense if

$$
\lim _{t \rightarrow \infty} \int_{t-T}^{t}\left\|e_{i}(\tau)\right\|^{2} d \tau=0, \quad i=1,2, \ldots, N
$$

and the system (1) is said to be globally asymptotically synchronized onto state $s(t)$ if

$$
\lim _{t \rightarrow \infty} e_{i}(t)=0, \quad i=1,2, \ldots, N .
$$

To achieve the control objective in (3) and (4), we need an adaptive control strategy to nodes in network (1). Then, the controlled network is given by

$$
\begin{aligned}
\dot{x}_{i}(t)= & f_{i}\left(x_{i}(t)\right)+\sum_{j=1}^{N} a_{i j} \Gamma g\left(x_{j}(t), \varphi_{i}(t)\right) \\
& +\sum_{j=1}^{N} b_{i j} \Gamma k\left(\dot{x}_{j}(t), \beta_{i}(t)\right) \\
& +u_{i}(t), \quad i=1,2, \ldots, N,
\end{aligned}
$$

where $u_{i}(t)=\left[u_{i 1}(t), u_{i 2}(t), \ldots, u_{i n}(t)\right]^{T} \in R^{n}, i=1,2, \ldots, N$, are the adaptive controllers to be designed. Since the row sum of coupling matrix is zero, it is easily obtained that $\sum_{j=1}^{N} a_{i j} \Gamma g\left(s(t), \varphi_{i}(t)\right)=\sum_{j=1}^{N} b_{i j} \Gamma k\left(\dot{s}(t), \beta_{i}(t)\right)=0$, which implies the dynamical error equations as

$$
\begin{aligned}
\dot{e}_{i}(t)= & \dot{x}_{i}(t)-\dot{s}(t)=f_{i}\left(x_{i}(t)\right) \\
& +\sum_{j=1}^{N} a_{i j} \Gamma\left(g\left(x_{j}(t), \varphi_{i}(t)\right)-g\left(s(t), \varphi_{i}(t)\right)\right) \\
& +\sum_{j=1}^{N} b_{i j} \Gamma\left(k\left(\dot{x}_{j}(t), \beta_{i}(t)\right)-k\left(\dot{s}(t), \beta_{i}(t)\right)\right) \\
& -\dot{s}(t)+u_{i}(t) .
\end{aligned}
$$

Our objective is to design a distributed adaptive control law $u_{i}(t)$ so as to obtain the convergence of the synchronization errors.

In order to derive the main results, the following assumptions and lemmas are introduced. 
Assumption 2 (Lipschits condition). For the system (1), there exist positive constants $l_{i}>0, i=1, \ldots, N$ such that

$$
\left\|f_{i}(x)-f_{i}(y)\right\| \leq l_{i}\|x-y\|, \quad \forall x, y \in R^{n}, 1 \leq i \leq N .
$$

Remark 3. For many well-known systems, such as Lorenz's system, Chen's system, and Lü's system, the above condition is satisfied.

Assumption 4 (see [33]). For the unknown continuous functions $g(\cdot, \cdot), k(\cdot, \cdot)$, the following inequality holds:

$$
\begin{aligned}
& \left\|g\left(x_{j}(t), \varphi_{i}(t)\right)-g\left(s(t), \varphi_{i}(t)\right)\right\|^{2} \\
& \quad \leq\left\|x_{j}(t)-s(t)\right\|^{2} h\left(x_{j}(t), s(t)\right) \lambda\left(\varphi_{i}(t)\right), \\
& \left|k\left(\dot{x}_{j}(t), \beta_{i}(t)\right)-k\left(\dot{s}(t), \beta_{i}(t)\right)\right| \\
& \quad \leq M\left(\beta_{i}(t)\right)\left|\dot{x}_{j}(t)-\dot{s}(t)\right| \triangleq M_{i}(t)\left|\dot{e}_{j}(t)\right|,
\end{aligned}
$$

where $h(\cdot, \cdot)$ is an known nonnegative continuous function $\lambda(\cdot)$ is a unknown nonnegative continuous function, $M(\cdot)$ or $M_{i}(\cdot)$ is an $n \times n$ unknown nonnegative continuous function matrix.

Remark 5. It is well known that the estimation of unknown nonlinear parameters in the systems is a difficult problem. In this paper, we separate the unknown parameters from the nonlinear function in (8), according to the separation principle in [33]; thus, Assumption 4 is reasonable and easily obtained. By using Assumption 4, we are able to solve the synchronization problem for a class of nonlinearly parameterized systems with nonlinear derivative couplings.

Assumption 6. In the given networks in (1), $\varphi_{i}(t), \beta_{i}(t)$ are unknown time-varying periodic functions with a known period $T$.

From Assumption 6, it is easy to see that $\lambda\left(\varphi_{i}(t)\right)$ and each element in $M_{i}(t)$ are periodic functions with the same period $T$. Suppose

$$
\lambda\left(\varphi_{i}(t)\right)=\phi_{i}(t)+\theta_{i},
$$

where $\phi_{i}(t)$ is an unknown continuous periodic function with a known period $T$ and $\theta_{i}$ is an unknown constant parameter.

Assumption 7. In network (1), the inner coupling matrix $\Gamma$ and $h(\cdot, \cdot)$ satisfies

$$
\begin{array}{r}
\max \left(\|\Gamma\|, \max \left(\left|\gamma_{i}\right|\right)\right) \leq \gamma, \quad h\left(x_{i}(t), s(t)\right)<H \\
i=1,2, \ldots, N
\end{array}
$$

where $\gamma, H$ are positive constants.

Assumption 8. Assume that the state and the state derivative of system (1) are measurable.

Remark 9. This assumption is necessary to design controller and adaptive laws. Assumption 8 seems to be restrictive. The observer for state derivative will be considered in the near future.
Lemma 10 (Young's inequality). For vectors $x, y \in R^{n}$, and any positive constant $c$, the following matrix inequality holds:

$$
x^{T} y \leq c x^{T} x+\frac{1}{4 c} y^{T} y
$$

Lemma 11. For any vectors $x, y \in R^{n}$, according to the properties of matrix trace, the following equality holds:

$$
x^{T} y=\operatorname{tr}\left(y x^{T}\right)=\operatorname{tr}\left(x y^{T}\right)
$$

Lemma 12 (Barbalat's Lemma [34]). If $e(t) \in L_{2}$ and $\dot{e}(t) \epsilon$ $L_{\infty}$, then $\lim _{t \rightarrow \infty} e(t)=0$, where $L_{2}=\{x \mid x:[0, \infty) \rightarrow$ $\left.R,\left(\int_{0}^{\infty}|x(t)|^{2}\right)^{1 / 2}<\infty\right\}$ and $L_{\infty}=\{x \mid x:[0, \infty) \rightarrow$ $\left.R, \sup _{t \geq 0}|x(t)|<\infty\right\}$.

Lemma 13. For a positive constant $T$, if $\forall t \geq 0, f(t)$ is a continuous function and $\int_{t-T}^{t} f(\tau) d \tau$ exists and is bounded with $M$. Then, $f(t)$ is bounded.

Proof. In fact, if $f(t)$ is unbounded, we can get $\forall N>0, \exists \tau \in$ $[t-T, t], f(\tau)>N$. As $f(t)$ is continuous function, according to the properties of the continuous functions, we have $\exists \varepsilon>$ $0 \forall k \in[\tau-\varepsilon, \tau+\varepsilon] \subset[t-T, t], f(k)>N$; then $\int_{t-T}^{t} f(\tau) d \tau \geq$ $\int_{\tau-\varepsilon}^{\tau+\varepsilon} f(\tau) d t \geq 2 \varepsilon N$. We can choose $N=(M / 2 \varepsilon)+1$; then $\int_{t-T}^{t} f(\tau) d \tau>M$; this is a contradiction with the assumption. The proof is completed.

\section{Adaptive Synchronization of the Complex Dynamical Networks}

In this section, distributed adaptive controller and distributed adaptive laws are designed to control the given system to synchronize the given trajectory $s(t)$, and the synchronization process is proved.

Considering the controlled networks, we design the controller by

$$
\begin{aligned}
u_{i}(t)= & \dot{s}(t)-f_{i}(s(t)) \\
& -\gamma H e_{i}(t) \sum_{j=1}^{N} a_{j i}^{2}\left(\widehat{\phi}_{j}(t)+\widehat{\theta}_{j}(t)\right) \\
& -\gamma \sum_{j=1}^{N}\left|b_{i j}\right| \operatorname{diag}_{1 \leq j \leq N}\left(\operatorname{sign}\left(e_{i j}(t)\right)\right) \widehat{M}_{i}(t)\left|\dot{e}_{j}(t)\right|,
\end{aligned}
$$

where $\gamma, H$ satisfy condition (10), $N$ is the node number, and $\widehat{\phi}_{i}(t)$, and $\widehat{\theta}_{i}(t)$, are $\widehat{M}_{i}(t)$ used to estimate $\phi_{i}(t), \theta_{i}, M_{i}(t)$. $\left|\dot{e}_{j}(t)\right|=\left(\left|\dot{e}_{j 1}(t)\right|, \ldots,\left|\dot{e}_{j n}(t)\right|\right)^{T}$.

The constant parameter distributed update law is designed as follows:

$$
\dot{\hat{\theta}}_{i}(t)=r_{i} \sum_{j=1}^{N} a_{i j}^{2} e_{j}^{T}(t) e_{j}(t)
$$


and the time-varying distributed periodic adaptive learning laws as

$$
\begin{aligned}
& \widehat{\phi}_{i}(t) \\
& = \begin{cases}0, & t \in[-T, 0) \\
q_{i 0}(t) \sum_{j=1}^{N} a_{i j}^{2} e_{j}^{T}(t) e_{j}(t), & t \in[0, T) \\
\widehat{\phi}_{i}(t-T)+q_{i} \sum_{j=1}^{N} a_{i j}^{2} e_{j}^{T}(t) e_{j}(t), & t \in[T,+\infty),\end{cases}
\end{aligned}
$$

$\widehat{M}_{i}(t)$

$$
= \begin{cases}0, & t \in[-T, 0) \\ f_{i 0}(t) \sum_{j=1}^{N}\left|b_{i j}\right|\left|e_{i}(t)\right|\left|\dot{e}_{j}^{T}(t)\right|, & t \in[0, T) \\ \widehat{M}_{i}(t-T)+f_{i} \sum_{j=1}^{N}\left|b_{i j}\right|\left|e_{i}(t)\right|\left|\dot{e}_{j}^{T}(t)\right|, & t \in[T,+\infty),\end{cases}
$$

where $r_{i}, q_{i}$, and $f_{i}$ are positive constants and $q_{i 0}(t), f_{i 0}(t)$ are continuous and strictly increasing nonnegative functions satisfying $q_{i 0}(0)=0, q_{i 0}(T)=q_{i}$, and $f_{i 0}(0)=0, f_{i 0}(T)=f_{i}$ which ensure that $\widehat{\phi}_{i}(t), \widehat{M}_{i}(t)$ are continuous in $t=i T$, $i=1,2, \ldots, N,\left|e_{j}(t)\right|=\left(\left|e_{j 1}(t)\right|, \ldots,\left|e_{j n}(t)\right|\right)^{T}$.

The following theorem will give a sufficient condition for the controlled network in (5) to be asymptotical synchronization.

Theorem 14. Under Assumptions 2-7, the control law (13) with the adaptive learning laws (14)-(16) guarantees the controlled networks in (5) achieve asymptotic synchronization in the $L_{T}^{2}$ norm sense. All closed-loop signals are bounded. Furthermore, if $k(\cdot, \cdot)$ is bounded function, we can obtain globally asymptotical synchronization.

Proof. Define a Lyapunov-Krasovskii-like function as follows:

$$
\begin{aligned}
& V(t)= \frac{1}{2} \sum_{i=1}^{N} e_{i}^{T} e_{i}+\frac{\gamma H}{2} \sum_{i=1}^{N} \int_{t-T}^{t} q_{i}^{-1} \widetilde{\phi}_{i}^{2}(\tau) d \tau \\
&+\frac{\gamma H}{2} \sum_{i=1}^{N} r_{i}^{-1}\left(\widetilde{\theta}_{i}(t)+L\right)^{2} \\
&+\frac{\gamma}{2} \sum_{i=1}^{N} \int_{t-T}^{t} f_{i}^{-1} \operatorname{tr}\left(\widetilde{M}_{i}(\tau) \widetilde{M}_{i}^{T}(\tau)\right) d \tau, \\
& t \in[0, \infty),
\end{aligned}
$$

where $\widetilde{\phi}_{i}(t)=\phi_{i}(t)-\widehat{\phi}_{i}(t), \widetilde{\theta}_{i}(t)=\theta_{i}-\widehat{\theta}_{i}(t), \widetilde{M}_{i}(t)=M_{i}(t)-$ $\widehat{M}_{i}(t)$, and $L$ is a sufficiently large positive constant which will be determined later.
It should be noted that we define $\phi_{i}(t)=\phi_{i}(0), M_{i}(t)=$ $M_{i}(0) t \in[-T, 0)$; then, from the adaptive laws (15) and (16), we get

$$
\begin{aligned}
\widetilde{\phi}_{i}^{2}(t-T) & =\phi_{i}^{2}(t-T)=\phi_{i}^{2}(0), \\
\widetilde{M}_{i}(t-T) \widetilde{M}_{i}^{T}(t-T) & =M_{i}(t-T) M_{i}^{T}(t-T) \\
& =M_{i}(0) M_{i}^{T}(0), \quad t \in[0, T] .
\end{aligned}
$$

Firstly, the finiteness property of $V(t)$ for the period $[0, T)$ is given.

Consider the system (6) and the proposed control laws (14)-(16); it can be seen that the right-hand side of (6) is continuous with respect to all arguments. According to the existence theorem of differential equation, (6) has unique solution in the interval $\left[0, T_{1}\right) \subset[0, T)$ with $0<T_{1} \leq$ $T$. This can guarantee the boundedness of $V(t)$ over $\left[0, T_{1}\right)$. Therefore, we need only focus in the interval $\left[T_{1}, T\right)$.

The derivative of $V(t)$ with respect to time is given by

$$
\begin{aligned}
\dot{V}(t)= & \sum_{i=1}^{N} e_{i}^{T}(t) \dot{e}_{i}(t) \\
& +\frac{\gamma H}{2} \sum_{i=1}^{N}\left[q_{i}^{-1} \widetilde{\phi}_{i}^{2}(t)-q_{i}^{-1} \widetilde{\phi}_{i}^{2}(t-T)\right] \\
& +\gamma H \sum_{i=1}^{N} r_{i}^{-1}\left(\widetilde{\theta}_{i}(t)+L\right) \dot{\tilde{\theta}}_{i}(t) \\
& +\frac{\gamma}{2} \sum_{i=1}^{N}\left[f_{i}^{-1} \operatorname{tr}\left(\widetilde{M}_{i}(t) \widetilde{M}_{i}^{T}(t)\right)\right. \\
& \left.\quad-f_{i}^{-1} \operatorname{tr}\left(\widetilde{M}_{i}(t-T) \widetilde{M}_{i}^{T}(t-T)\right)\right] .
\end{aligned}
$$

Let us introduce some notations as

$$
\begin{gathered}
f_{i}\left(x_{i}(t)\right)-f_{i}(s(t))=\Phi, \\
g\left(x_{j}(t), \varphi_{i}(t)\right)-g\left(s(t), \varphi_{i}(t)\right)=\Lambda, \\
k\left(\dot{x}_{j}(t), \beta_{i}(t)\right)-k\left(\dot{s}_{j}(t), \beta_{i}(t)\right)=K .
\end{gathered}
$$

From (6) and (13), the first term on the right hand side of (19) satisfies

$$
\begin{gathered}
\sum_{i=1}^{N} e_{i}^{T}(t) \dot{e}_{i}(t) \\
=\sum_{i=1}^{N} e_{i}^{T}(t)\left[f_{i}\left(x_{i}(t)\right)+\sum_{j=1}^{N} a_{i j} \Gamma \Lambda\right. \\
\left.+\sum_{j=1}^{N} b_{i j} \Gamma K-\dot{s}(t)+u_{i}(t)\right] \\
=\sum_{i=1}^{N}\left(e_{i}^{T} \Phi+\sum_{j=1}^{N} a_{i j} e_{i}^{T} \Gamma \Lambda\right. \\
+\sum_{j=1}^{N} b_{i j} e_{i}^{T} \Gamma K-\gamma H \sum_{j=1}^{N} a_{j i}^{2}\left(\widehat{\phi}_{j}(t)+\widehat{\theta}_{j}(t)\right) e_{i}^{T} e_{i} \\
\left.-\gamma \sum_{j=1}^{N}\left|b_{i j}\right|\left|e_{i}^{T}(t)\right| \widehat{M}_{i}(t)\left|\dot{e}_{j}(t)\right|\right) .
\end{gathered}
$$


According to Assumptions 2-7 and Lemma 10, from the above equation, we get

$$
\begin{gathered}
\sum_{i=1}^{N} e_{i}^{T}(t) \dot{e}_{i}(t) \\
\leq \sum_{i=1}^{N}\left[c e_{i}^{T} e_{i}+\frac{1}{4 c} \phi^{T} \phi\right. \\
+\sum_{j=1}^{N}\left(d e_{i}^{T} e_{i}+\frac{1}{4 d} a_{i j}^{2} \Lambda^{T} \Gamma^{T} \Gamma \Lambda\right) \\
+\gamma \sum_{j=1}^{N}\left|b_{i j}\right|\left|e_{i}^{T}\right||K| \\
-\gamma H \sum_{j=1}^{N} a_{j i}^{2}\left(\widehat{\phi}_{j}(t)+\widehat{\theta}_{j}(t)\right) e_{i}^{T} e_{i} \\
\left.-\gamma \sum_{j=1}^{N}\left|b_{i j}\right|\left|e_{i}^{T}(t)\right| \widehat{M}_{i}(t)\left|\dot{e}_{j}(t)\right|\right] \\
\leq \sum_{i=1}^{N}\left[\left(c+\frac{l_{i}^{2}}{4 c}\right) e_{i}^{T} e_{i}+N d e_{i}^{T} e_{i}\right. \\
+\sum_{j=1}^{N} a_{i j}^{2}\left(\phi_{i}(t)+\theta_{i}\right) \frac{H \gamma^{2}}{4 d} e_{j}^{T} e_{j} \\
\left.+\gamma \sum_{j=1}^{N}\left|b_{i j}\right|\left|e_{i j}^{T}\right|\left|e_{i}^{T}(t)\right| \widehat{M}_{i}(t)\left|\dot{e}_{j}(t)\right|\right] \\
-\gamma H \sum_{j=1}^{N} a_{j i}^{2}(t)\left|\hat{e}_{j}(t)\right|
\end{gathered}
$$

where $c, d$ are positive constants. Choosing $c=1 / 2, d=\gamma / 4$, and according to Lemma 11, we have

$$
\begin{aligned}
& \sum_{i=1}^{N} e_{i}^{T}(t) \dot{e}_{i}(t) \\
& \leq \sum_{i=1}^{N}\left[\left(\frac{l_{i}}{2}+\frac{1}{2}+\frac{N \gamma}{4}\right.\right. \\
& \left.+\gamma H \sum_{j=1}^{N} a_{j i}^{2}\left(\widetilde{\phi}_{j}(t)+\widetilde{\theta}_{j}(t)\right)\right) e_{i}^{T} e_{i} \\
& \left.+\gamma \sum_{j=1}^{N}\left|b_{i j}\right|\left|e_{i}^{T}(t)\right| \widetilde{M}_{i}(t)\left|\dot{e}_{j}(t)\right|\right]
\end{aligned}
$$

$$
\begin{gathered}
=\sum_{i=1}^{N}\left[\left(\frac{l_{i}}{2}+\frac{1}{2}+\frac{N \gamma}{4}\right.\right. \\
\left.+\gamma H \sum_{j=1}^{N} a_{j i}^{2}\left(\widetilde{\phi}_{j}(t)+\widetilde{\theta}_{j}(t)\right)\right) e_{i}^{T} e_{i} \\
\left.+\gamma \operatorname{tr}\left(\sum_{j=1}^{N}\left|b_{i j}\right| \widetilde{M}_{i}(t)\left|\dot{e}_{j}(t)\right|\left|e_{i}^{T}(t)\right|\right)\right] \\
=\sum_{i=1}^{N}\left[\left(\frac{l_{i}}{2}+\frac{1}{2}+\frac{N \gamma}{4}\right.\right. \\
\left.+\sum_{j=1}^{N} a_{j i}^{2} \gamma H\left(\widetilde{\phi}_{j}(t)+\widetilde{\theta}_{j}(t)\right)\right) e_{i}^{T} e_{i} \\
\left.+\gamma \operatorname{tr}\left(\sum_{j=1}^{N}\left|b_{i j}\right|\left|e_{i}(t)\right|\left|\dot{e}_{j}^{T}(t)\right| \widetilde{M}_{i}^{T}(t)\right)\right] .
\end{gathered}
$$

Applying (14), the third term on the right-hand side of (19) satisfies

$$
\begin{aligned}
& \gamma H \sum_{i=1}^{N} r_{i}^{-1}\left(\widetilde{\theta}_{i}(t)+L\right) \dot{\tilde{\theta}}_{i}(t) \\
& \quad=-\gamma H \sum_{i=1}^{N}\left(\widetilde{\theta}_{i}(t)+L\right) \sum_{j=1}^{N} a_{i j}^{2} e_{j}^{T} e_{j} .
\end{aligned}
$$

Let us focus on the second and fourth terms on the righthand side of (19). In the interval $\left[T_{1}, T\right)$, since $q_{i 0}(t), f_{i 0}(t)$ are continuous and strictly increasing functions, $q_{i}^{-1} \leq q_{i 0}^{-1}(t)<$ $\infty, f_{i}^{-1} \leq f_{i 0}^{-1}(t)<\infty$ are ensured, we obtain

$$
\begin{aligned}
& \frac{\gamma H}{2} \sum_{i=1}^{N}\left[q_{i}^{-1} \widetilde{\phi}_{i}^{2}(t)-q_{i}^{-1} \widetilde{\phi}_{i}^{2}(t-T)\right] \\
& =\frac{\gamma H}{2} \sum_{i=1}^{N}\left[q_{i}^{-1} \widetilde{\phi}_{i}^{2}(t)-q_{i}^{-1} \widetilde{\phi}_{i}^{2}(0)\right] \\
& \leq \frac{\gamma H}{2} \sum_{i=1}^{N} q_{i 0}^{-1} \widetilde{\phi}_{i}^{2}(t) \\
& =\frac{\gamma H}{2} \sum_{i=1}^{N} q_{i 0}^{-1}\left[\phi_{i}^{2}(t)+2 \widehat{\phi}_{i}^{2}(t)\right. \\
& \leq \frac{\gamma H}{2} \sum_{i=1}^{N} q_{i 0}^{-1}\left[\phi_{i}^{2}(t)+2 \widehat{\phi}_{i}^{2}(t)\right. \\
& \left.\quad-2 \phi_{i}(t) \widehat{\phi}_{i}(t)\right] \\
& =\frac{\gamma H}{2} \sum_{i=1}^{N} q_{i 0}^{-1} \phi_{i}^{2}(t)-\gamma H \sum_{i=1}^{N} \sum_{j=1}^{N} a_{i j}^{2} \widetilde{\phi}_{i}(t) e_{j}^{T} e_{j} .
\end{aligned}
$$


According to Lemma 11, we can get

$$
\begin{aligned}
\operatorname{tr} & \left(\widetilde{M}_{i}(t) \widetilde{M}_{i}^{T}(t)\right) \\
& =\operatorname{tr}\left[\left(M_{i}-\widehat{M}_{i}(t)\right)\left(M_{i}-\widehat{M}_{i}(t)\right)^{T}\right] \\
& =\operatorname{tr}\left(M_{i} M_{i}^{T}-M_{i} \widehat{M}_{i}^{T}-\widehat{M}_{i} M_{i}^{T}+\widehat{M}_{i} \widehat{M}_{i}^{T}\right) \\
& =\operatorname{tr}\left(M_{i} M_{i}^{T}+2 \widehat{M}_{i} \widehat{M}_{i}^{T}-M_{i} \widehat{M}_{i}^{T}-\widehat{M}_{i} M_{i}^{T}-\widehat{M}_{i} \widehat{M}_{i}^{T}\right) \\
& \leq \operatorname{tr}\left(M_{i} M_{i}^{T}+2 \widehat{M}_{i} \widehat{M}_{i}^{T}-M_{i} \widehat{M}_{i}^{T}-\widehat{M}_{i} M_{i}^{T}\right) \\
& =\operatorname{tr}\left(M_{i} M_{i}^{T}\right)+2 \operatorname{tr}\left(\widehat{M}_{i} \widehat{M}_{i}^{T}-M_{i} \widehat{M}_{i}^{T}\right) \\
& =\operatorname{tr}\left(M_{i} M_{i}^{T}\right)-2 \operatorname{tr}\left(\widetilde{M}_{i} \widehat{M}_{i}^{T}\right) .
\end{aligned}
$$

Then from (26), the last term on the right-hand side of (19) satisfies

$$
\begin{aligned}
\frac{\gamma}{2} \sum_{i=1}^{N}[ & f_{i}^{-1} \operatorname{tr}\left(\widetilde{M}_{i}(t) \widetilde{M}_{i}^{T}(t)\right) \\
& \left.-f_{i}^{-1} \operatorname{tr}\left(\widetilde{M}_{i}(t-T) \widetilde{M}_{i}^{T}(t-T)\right)\right] \\
\leq & \frac{\gamma}{2} \sum_{i=1}^{N} f_{i}^{-1} \operatorname{tr}\left(\widetilde{M}_{i}(t) \widetilde{M}_{i}^{T}(t)\right) \\
\leq & \frac{\gamma}{2} \sum_{i=1}^{N} f_{i 0}^{-1}\left[\operatorname{tr}\left(M_{i} M_{i}^{T}\right)-2 \operatorname{tr}\left(\widetilde{M}_{i} \widehat{M}_{i}^{T}\right)\right] \\
= & \frac{\gamma}{2} \sum_{i=1}^{N} f_{i 0}^{-1} \operatorname{tr}\left(M_{i} M_{i}^{T}\right)-\gamma \sum_{i=1}^{N} \operatorname{tr}\left(f_{i 0}^{-1} \widetilde{M}_{i} \widehat{M}_{i}^{T}\right) \\
= & \frac{\gamma}{2} \sum_{i=1}^{N} f_{i 0}^{-1} \operatorname{tr}\left(M_{i} M_{i}^{T}\right) \\
& -\gamma \sum_{i=1}^{N} \operatorname{tr}\left(\sum_{j=1}^{N}\left|b_{i j}\right|\left|e_{i}(t)\right|\left|\dot{e}_{j}^{T}(t)\right| \widetilde{M}_{i}^{T}(t)\right) .
\end{aligned}
$$

Substituting (23)-(27) into (19) we obtain

$$
\begin{aligned}
\dot{V}(t) \leq & \sum_{i=1}^{N}\left(\frac{l_{i}}{2}+\frac{1}{2}+\frac{N \gamma}{4}-L \sum_{j=1}^{N} a_{j i}^{2}\right) e_{i}^{T} e_{i} \\
& +\frac{\gamma H}{2} \sum_{i=1}^{N} q_{i 0}^{-1}(t) \phi_{i}^{2}(t)+\frac{\gamma}{2} \sum_{i=1}^{N} f_{i 0}^{-1} \operatorname{tr}\left(M_{i} M_{i}^{T}\right)
\end{aligned}
$$

It is obvious that there exist sufficiently large positive constants $L$ such that

$$
\frac{1}{2}+\frac{1}{2} l_{i}^{2}+\frac{N \gamma}{4}-L \sum_{j=1}^{N} a_{j i}^{2}<0, \quad i=1, \ldots, N
$$

According to (28) we have

$$
\begin{array}{r}
\dot{V}(t) \leq \frac{\gamma H}{2} \sum_{i=1}^{N} q_{i 0}^{-1}(t) \phi_{i}^{2}(t)+\frac{\gamma}{2} \sum_{i=1}^{N} f_{i 0}^{-1} \operatorname{tr}\left(M_{i} M_{i}^{T}\right), \\
t \in\left[T_{1}, T\right) .
\end{array}
$$

For $\forall t \in\left[T_{1}, T\right)$, since $\phi_{i}(t)$ is continuous and periodic and every element in matrix $M_{i}(\cdot)$ is continuous function, the boundedness of them can be obtained. The boundedness of $\phi_{i}(t)$ and $\operatorname{tr}\left(M_{i} M_{i}^{T}\right)$ leads to the boundedness of $\dot{V}(t)$. That is, $V(t)$ is bounded in $[0, T)$. For $V\left(T_{1}\right)$ is bounded, the finiteness of $V(t)$ is obvious by using integral technique, $\forall t \in[0, T)$.

Next step, the asymptotical convergence of $e(t)$ is proved. According to (17), $\forall t \geq T$, we can get

$$
\begin{aligned}
& \Delta V(t)= V(t)-V(t-T) \\
&=-\frac{1}{2} \sum_{i=1}^{N} e_{i}^{T}(t) e_{i}(t) \\
&-\frac{1}{2} \sum_{i=1}^{N} e_{i}^{T}(t-T) e_{i}(t-T) \\
&+\frac{\gamma H}{2} \sum_{i=1}^{N} r_{i}^{-1}\left(\widetilde{\theta}_{i}(t)+L\right)^{2} \\
&-\frac{\gamma H}{2} \sum_{i=1}^{N} r_{i}^{-1}\left(\widetilde{\theta}_{i}(t-T)+L\right)^{2} \\
&+\frac{\gamma H}{2} \sum_{i=1}^{N} \int_{t-T}^{t}\left[q_{i}^{-1} \widetilde{\phi}_{i}^{2}(\tau)-q_{i}^{-1} \widetilde{\phi}_{i}^{2}(\tau-T)\right] d \tau \\
&+\frac{\gamma}{2} \sum_{i=1}^{N} \int_{t-T}^{t} f_{i}^{-1}\left[\operatorname{tr}\left(\widetilde{M}_{i}(\tau) \widetilde{M}_{i}^{T}(\tau)\right)\right. \\
&\left.-\operatorname{tr}\left(\widetilde{M}_{i}(\tau-T) \widetilde{M}_{i}^{T}(\tau-T)\right)\right] d \tau .
\end{aligned}
$$

With Newton-Leibniz's formula, the first two terms on the right-hand side of (31) satisfied

$$
\begin{aligned}
& \frac{1}{2} \sum_{i=1}^{N} e_{i}^{T}(t) e_{i}(t)-\frac{1}{2} \sum_{i=1}^{N} e_{i}^{T}(t-T) e_{i}(t-T) \\
& =\sum_{i=1}^{N} \int_{t-T}^{t} e_{i}^{T}(\tau) \dot{e}_{i}(\tau) d \tau \\
& \leq \sum_{i=1}^{N} \int_{t-T}^{t}\left(\frac{1}{2}+\frac{1}{2} l_{i}^{2}+\frac{N \gamma}{4}\right) e_{i}^{T}(\tau) e_{i}(\tau) d \tau \\
& \quad+\sum_{i=1}^{N} \sum_{j=1}^{N} \int_{t-T}^{t} a_{j i}^{2} \gamma H e_{i}^{T}(\tau) e_{i}(\tau)\left(\widetilde{\phi}_{j}(\tau)+\widetilde{\theta}_{j}(\tau)\right) d \tau \\
& \quad+\sum_{i=1}^{N} \int_{t-T}^{t}\left|b_{i j}\right| \gamma \operatorname{tr}\left(\sum_{j=1}^{N}\left|e_{i}(\tau)\right|\left|\dot{e}_{j}^{T}(\tau)\right| \widetilde{M}_{i}^{T}(\tau)\right) d \tau .
\end{aligned}
$$


Using the algebraic relation

$$
\begin{gathered}
(a-b)^{T} G(a-b)-(a-c)^{T} G(a-c) \\
=(c-b)^{T} G[2(a-b)+(b-c)],
\end{gathered}
$$

where $a, b, c \in R^{p}, G \in R^{p \times p}$.

After choosing $G=1, a=\phi_{i}(\tau), b=\widehat{\phi}(\tau), a-b=\widetilde{\phi}_{i}(\tau)$, $\phi_{i}(\tau)=\phi_{i}(\tau-T), c=\widehat{\phi}_{i}(\tau-T)$, and $a-c=\widetilde{\phi}_{i}(\tau-T)$, we can get

$$
\begin{aligned}
\frac{\gamma H}{2} \sum_{i=1}^{N} q_{i}^{-1} \widetilde{\phi}_{i}^{2}(\tau)-\frac{\gamma H}{2} \sum_{i=1}^{N} q_{i}^{-1} \widetilde{\phi}_{i}^{2}(\tau-T) \\
=\frac{\gamma H}{2} \sum_{i=1}^{N} q_{i}^{-1}\left[2 \widetilde{\phi}_{i}(\tau)+\widehat{\phi}_{i}(\tau)-\widehat{\phi}_{i}(\tau-T)\right] \\
\quad \times\left[\widehat{\phi}_{i}(\tau-T)-\widehat{\phi}_{i}(\tau)\right] \\
=-\gamma H \sum_{i=1}^{N}\left(\widetilde{\phi}_{i}(\tau)+\frac{1}{2} q_{i} \sum_{j=1}^{N} a_{i j}^{2} e_{j}^{T} e_{j}\right) \sum_{k=1}^{N} a_{i k}^{2} e_{k}^{T} e_{k} .
\end{aligned}
$$

From Lemma 11, we have

$$
\begin{aligned}
& \operatorname{tr}\left(\widetilde{M}_{i}(\tau) \widetilde{M}_{i}^{T}(\tau)\right)-\operatorname{tr}\left(\widetilde{M}_{i}(\tau-T) \widetilde{M}_{i}^{T}(\tau-T)\right) \\
&= \operatorname{tr}\left(\widetilde{M}_{i}(\tau) \widetilde{M}_{i}^{T}(\tau)-\widetilde{M}_{i}(\tau-T) \widetilde{M}_{i}^{T}(\tau)\right. \\
&\left.+\widetilde{M}_{i}(\tau-T) \widetilde{M}_{i}^{T}(\tau)-\widetilde{M}_{i}(\tau-T) \widetilde{M}_{i}^{T}(\tau-T)\right) \\
&=\operatorname{tr}\left(\left[\widetilde{M}_{i}(\tau)-\widetilde{M}_{i}(\tau-T)\right] \widetilde{M}_{i}^{T}(\tau)\right. \\
&\left.\quad+\widetilde{M}_{i}(\tau-T)\left[\widetilde{M}_{i}^{T}(\tau)-\widetilde{M}_{i}^{T}(\tau-T)\right]\right) \\
&=\operatorname{tr}\left(\left[\widetilde{M}_{i}(\tau)-\widetilde{M}_{i}(\tau-T)\right]\left[\widetilde{M}_{i}^{T}(\tau)+\widetilde{M}_{i}^{T}(\tau-T)\right]\right) \\
&=\operatorname{tr}\left(\left[\widetilde{M}_{i}(\tau)-\widetilde{M}_{i}(\tau-T)\right]\right. \\
&\left.\quad \times\left[2 \widetilde{M}_{i}^{T}(\tau)+\widetilde{M}_{i}^{T}(\tau-T)-\widetilde{M}_{i}^{T}(\tau)\right]\right) .
\end{aligned}
$$

According to (34) and (35), the last two terms on the right-hand side of (31) satisfy

$$
\begin{aligned}
& \frac{\gamma H}{2} \sum_{i=1}^{N} \int_{t-T}^{t}\left[q_{i}^{-1} \widetilde{\phi}_{i}^{2}(\tau)-q_{i}^{-1} \widetilde{\phi}_{i}^{2}(\tau-T)\right] d \tau \\
& =-\gamma H \sum_{i=1}^{N} \int_{t-T}^{t}\left[\widetilde{\phi}_{i}(\tau)+\frac{1}{2} q_{i} \sum_{j=1}^{N} a_{i j}^{2} e_{j}^{T} e_{j}\right] \\
& \quad \times \sum_{k=1}^{N} a_{i k}^{2} e_{k}^{T} e_{k} d \tau,
\end{aligned}
$$

$$
\begin{aligned}
& \frac{\gamma}{2} \sum_{i=1}^{N} \int_{t-T}^{t} f_{i}^{-1} \\
& \times\left[\operatorname{tr}\left(\widetilde{M}_{i}(\tau) \widetilde{M}_{i}^{T}(\tau)\right)\right. \\
& \left.-\operatorname{tr}\left(\widetilde{M}_{i}(\tau-T) \widetilde{M}_{i}^{T}(\tau-T)\right)\right] d \tau \\
& =\frac{\gamma}{2} \sum_{i=1}^{N} \int_{t-T}^{t} f_{i}^{-1} \\
& \times \operatorname{tr}\left[\left(f_{i} \sum_{j=1}^{N}\left|b_{i j}\right|\left|e_{i}\right|\left|\dot{e}_{j}^{T}\right|\right)\right. \\
& \left.\times\left(2 \widetilde{M}_{i}^{T}(\tau)-\left(f_{i} \sum_{j=1}^{N}\left|b_{i j}\right|\left|e_{i}\right|\left|\dot{e}_{j}^{T}\right|\right)^{T}\right)\right] d \tau \\
& =-\gamma \sum_{i=1}^{N} \int_{t-T}^{t} \operatorname{tr}\left(\sum_{j=1}^{N}\left|b_{i j}\right|\left|e_{i}\right|\left|\dot{e}_{j}^{T}\right| \widetilde{M}_{i}^{T}(\tau)\right) d \tau \\
& -\frac{\gamma}{2} \sum_{i=1}^{N} \int_{t-T}^{t} \sum_{i=1}^{N} f_{i} \operatorname{tr}\left[\left(\sum_{j=1}^{N}\left|b_{i j}\right|\left|e_{i}\right|\left|\dot{e}_{j}^{T}\right|\right)\right. \\
& \left.\times\left(\sum_{j=1}^{N}\left|b_{i j}\right|\left|e_{i}\right|\left|\dot{e}_{j}^{T}\right|\right)^{T}\right] d \tau .
\end{aligned}
$$

The other terms of right-hand side of (31) can be simplified as follows:

$$
\begin{aligned}
& \frac{\gamma H}{2} \sum_{i=1}^{N} r_{i}^{-1}\left(\widetilde{\theta}_{i}(t)+L\right)^{2}-\frac{\gamma H}{2} \sum_{i=1}^{N} r_{i}^{-1}\left(\widetilde{\theta}_{i}(t-T)+L\right)^{2} \\
& \quad=\gamma H \sum_{i=1}^{N} \int_{t-T}^{t} r_{i}^{-1}\left(\widetilde{\theta}_{i}(\tau)+L\right) \dot{\tilde{\theta}}_{i}(\tau) d \tau \\
& \quad=-\gamma H \sum_{i=1}^{N} \int_{t-T}^{t}\left(\widetilde{\theta}_{i}(\tau)+L\right) \sum_{j=1}^{N} a_{i j}^{2} e_{j}^{T}(\tau) e_{j}(\tau) d \tau .
\end{aligned}
$$

Substituting (32) and (36)-(38) into (31), we can attain

$$
\Delta V(t) \leq \sum_{i=1}^{N} \int_{t-T}^{t}\left(\frac{1}{2}+\frac{1}{2} l_{i}^{2}+\frac{N \gamma}{4}-L \sum_{j=1}^{N} a_{j i}^{2}\right) e_{i}^{T}(\tau) e_{i}(\tau) d \tau .
$$

Choosing $1 / 2+(1 / 2) l_{i}^{2}+N \gamma / 4-L \sum_{j=1}^{N} a_{j i}^{2}<0, i=$ $1, \ldots, N$, we can obtain

$$
\Delta V(t)<0 .
$$

For any $t \in[l T,(l+1) T], l=1,2, \ldots$, and denoting $t_{0}=$ $t-l T, t_{0} \in[0, T)$, we have

$$
V(t)=V\left(t_{0}\right)+\sum_{j=0}^{l-1} \Delta V(t-j T) .
$$


Considering $t_{0} \in[0, T)$ and the positive of $V(t)$, according to (41), we obtain

$$
\begin{gathered}
V(t)<\max _{t_{0} \in[0, T)} V\left(t_{0}\right) \\
-\sum_{j=0}^{l-1} \sum_{i=1}^{N} \int_{t-(j+1) T}^{t-j T}\left(L \sum_{j=1}^{N} a_{j i}^{2}-\frac{1}{2}-\frac{1}{2} l_{i}^{2}-\frac{N \gamma}{4}\right) \\
\quad \times e_{i}^{T}(\tau) e_{i}(\tau) d \tau .
\end{gathered}
$$

Since $V\left(t_{0}\right)$ is bounded in the interval $[0, T)$, according to the convergence theorem of the sum of series and (42), the error $e(t)$ converges to zero asymptotically in $L_{T}^{2}$ norm. That is to say, we have

$$
\lim _{t \rightarrow \infty} \int_{t-T}^{t} e_{i}^{T}(\tau) e_{i}(\tau) d \tau=0
$$

Finally, we prove that all the closed-loop signals are bounded. Considering $\forall t \in[T, \infty)$, the derivative of $V(t)$ is

$$
\begin{aligned}
& \dot{V}(t)= \sum_{i=1}^{N} e_{i}^{T}(t) \dot{e}_{i}(t)+\frac{\gamma H}{2} \sum_{i=1}^{N} q_{i}^{-1} \widetilde{\phi}_{i}^{2}(t) \\
&-\frac{\gamma H}{2} \sum_{i=1}^{N} q_{i}^{-1} \widetilde{\phi}_{i}^{2}(t-T) \\
&+\gamma H \sum_{i=1}^{N} r_{i}^{-1}\left(\widetilde{\theta}_{i}(t)+L\right) \dot{\tilde{\theta}}_{i}(t) \\
&+\frac{\gamma}{2} f_{i}^{-1}\left[\operatorname{tr}\left(\widetilde{M}_{i}(t) \widetilde{M}_{i}^{T}(t)\right)\right. \\
& \leq-\frac{\gamma H}{2} \sum_{i=1}^{N} q_{i}\left(\sum_{j=1}^{N} a_{i j}^{2} e_{j}^{T} e_{j}\right)^{2} \\
&-\sum_{i=1}^{N}\left(L \sum_{j=1}^{N} a_{j i}^{2}-\frac{1}{2}-\frac{1}{2} l_{i}^{2}-\frac{N \gamma}{4}\right) e_{i}^{T} e_{i} \\
&-\frac{\gamma}{2} \sum_{i=1}^{N} f_{i} \operatorname{tr}\left[\left(\sum_{j=1}^{N}\left|b_{i j}\right|\left|e_{i}\right|\left|\dot{e}_{j}^{T}\right|\right)\right. \\
& \times\left(\sum_{j=1}^{N}\left|b_{i j}\right|\left|e_{i}\right|\left|\dot{e}_{j}^{T}\right|\right)
\end{aligned}
$$

Having the two sides of (44) integration, one can obtain

$$
\begin{aligned}
V(t) \leq & V(T)-\frac{\gamma H}{2} \sum_{i=1}^{N} \int_{T}^{t} q_{i}\left(\sum_{j=1}^{N} a_{i j}^{2} e_{j}^{T} e_{j}\right)^{2} d \tau \\
& -\sum_{i=1}^{N} \int_{T}^{t}\left(L \sum_{j=1}^{N} a_{j i}^{2}-\frac{1}{2}-\frac{1}{2} l_{i}^{2}-\frac{N \gamma}{4}\right) e_{i}^{T} e_{i} d \tau
\end{aligned}
$$

$$
\begin{aligned}
-\frac{\gamma}{2} \sum_{i=1}^{N} f_{i} \int_{t-T}^{t} \operatorname{tr} & {\left[\left(\sum_{j=1}^{N}\left|b_{i j}\right|\left|e_{i}\right|\left|\dot{e}_{j}^{T}\right|\right)\right.} \\
& \left.\times\left(\sum_{j=1}^{N}\left|b_{i j}\right|\left|e_{i}\right|\left|\dot{e}_{j}^{T}\right|\right)^{T}\right] d \tau .
\end{aligned}
$$

Choosing $\left(L>1 / 2+\left((1 / 2) l_{i}^{2}\right)+N \gamma / 4\right) / \sum_{j=1}^{N} a_{j i}^{2}, i=$ $1, \ldots, N$, we have

$$
V(t)<V(T)
$$

From the boundedness of $V(t)$ and (17), we can conclude that $e_{i}, \int_{t-T}^{t} \widetilde{\phi}_{i}^{2}(\tau) d \tau, \widetilde{\theta}_{i}(t)$ For $\theta_{i}$ is constant and $\int_{t-T}^{t} \operatorname{tr}\left(\widetilde{M}_{i}(\tau) \widetilde{M}_{i}^{T}(\tau)\right) d \tau$ are all bounded., it implies the boundedness of $\widehat{\theta}_{i}(t)$. According to Lemma 13 and the continuity of $\widetilde{\phi}_{i}(t)$ and $\widetilde{M}_{i}(t)$, the boundedness of $\widetilde{\phi}_{i}(t)$ and $\widetilde{M}_{i}(t)$ are obviously obtained. As $\phi_{i}(t)$ is a continuous periodic function and $M_{i}(t)$ is continuous periodic matrix, we can get that $\widehat{\phi}_{i}(t), \widehat{M}_{i}(t)$ are bounded. According to (1), the boundedness of the control input $u_{i}(t)$ is obtained. Since $e_{i}(t)$ is bounded, the boundedness of $x_{i}(t)$ is received. The proof is completed.

It is worth mentioning that when $k(\cdot, \cdot)$ is bounded function, the boundedness of $\dot{e}_{i}(t)$ can be easy to get. Then, from Lemma 12 we can obtain the error globally asymptotical synchronization.

\section{Simulation Example}

To demonstrate the theoretical result obtained in Section 3, the following dynamical network with six non-identical nodes is considered:

$$
\left.\begin{array}{rl}
\dot{x}_{i}(t)= & f_{i}\left(x_{i}(t)\right) \\
& +\sum_{j=1}^{N} a_{i j} \Gamma \exp \left(-\varphi_{i}(t)\left(\begin{array}{c}
x_{j 1}^{2}(t) \\
x_{j 2}^{2}(t) \\
x_{j 3}^{2}(t)
\end{array}\right)\right)
\end{array}\right)
$$



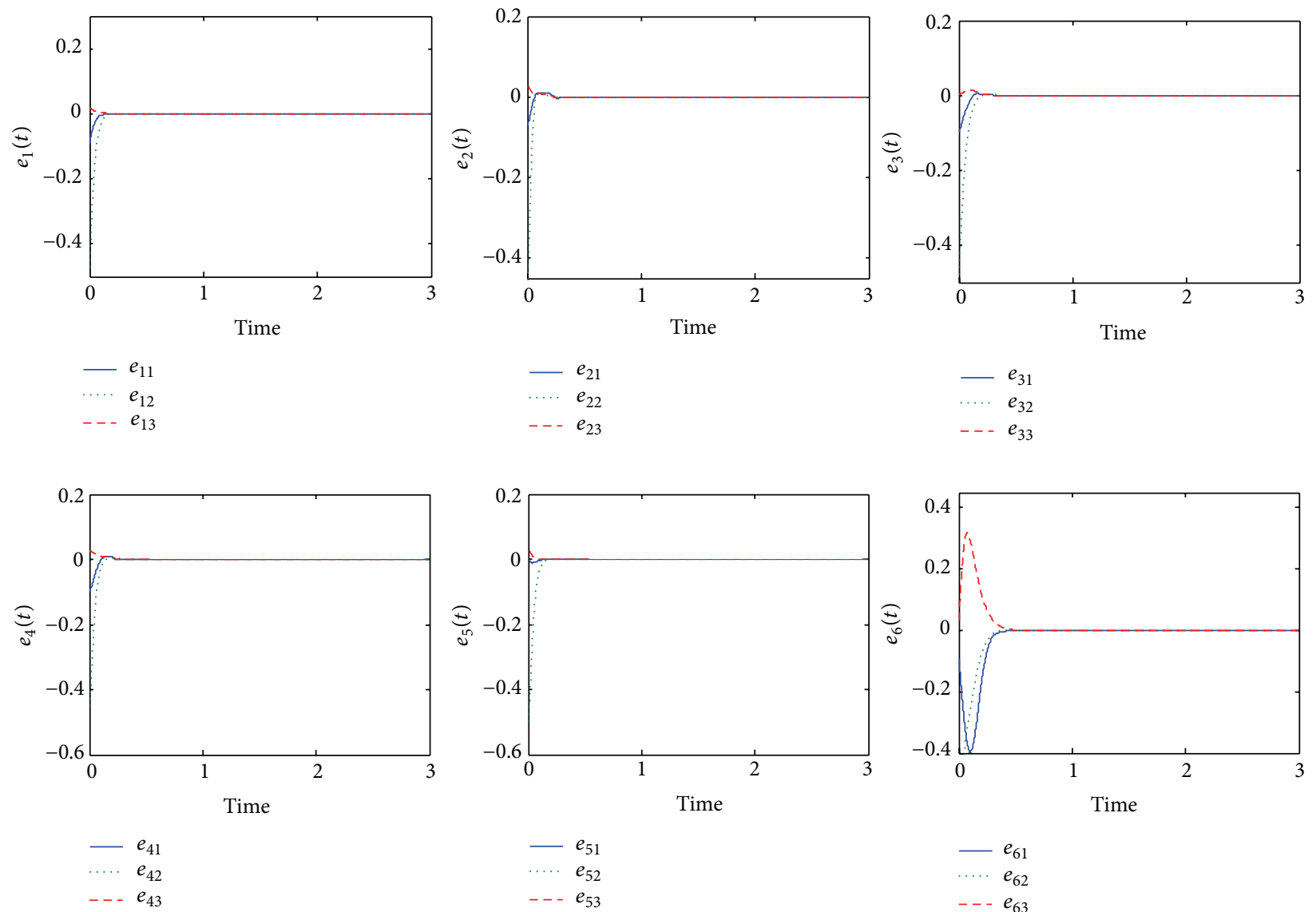

FIGURE 1: The evaluations of synchronization errors $e_{i}(t), i=1, \ldots, 6$.

where

$$
\begin{aligned}
& f_{1}\left(x_{1}\right)=\left(\begin{array}{c}
-2.5 x_{11}+0.3 x_{12}+0.9 x_{13}+3 x_{13}^{2} \\
0.6 x_{11}-2.6 x_{12}+3 x_{12}^{2}+x_{13} \\
-2.8 x_{11}+\sin x_{11} \cos x_{12}-2.2 x_{13}
\end{array}\right) \\
& f_{2}\left(x_{2}\right)=\left(\begin{array}{c}
-2.5 x_{21}+x_{22}+x_{21} x_{22}+x_{23} \\
0.5 x_{21}-0.8 x_{22}-\sin x_{22}+x_{23}-x_{23}^{2} \\
-2 x_{21}-0.8 x_{23}-0.5 \sin \left(2 x_{23}\right)
\end{array}\right) \\
& f_{3}\left(x_{3}\right)=\left(\begin{array}{c}
-2.5 x_{31}+x_{31} \sin x_{31}+x_{32}+1.4 x_{33} \\
0.5 x_{31}-2.5 x_{32}+x_{33} \\
-2 x_{31}+x_{32} x_{33}-0.6 x_{33}-x_{33} \cos x_{33}
\end{array}\right), \\
& f_{4}\left(x_{4}\right)=\left(\begin{array}{c}
-2.5 x_{41}+1.5 x_{42}+1.5 x_{43} \\
x_{41}-2.5 x_{42}+x_{42}^{2}+1.5 x_{43}+x_{43}^{2} \\
-2 x_{41}+x_{41} x_{43}-2.5 x_{43}
\end{array}\right) \\
& f_{5}\left(x_{5}\right)=\left(\begin{array}{c}
-2.1 x_{51}+0.5 x_{51}^{2}+1.4 x_{52}+x_{53} \\
0.9 x_{51}-2.1 x_{52}+x_{52} x_{53}+x_{53} \\
-2 x_{51}+0.4 x_{52}-2.1 x_{53}
\end{array}\right) \\
& f_{6}\left(x_{6}\right)=\left(\begin{array}{c}
-3.2 x_{61}+10 x_{62}+2.95\left(\left|x_{61}+1\right|-\left|x_{61}-1\right|\right) \\
x_{61}-x_{62}+x_{63} \\
-14.7 x_{62}
\end{array}\right)
\end{aligned}
$$$$
\begin{gathered}
\Gamma=\left[\begin{array}{lll}
1 & 0 & 0 \\
0 & 1 & 0 \\
0 & 0 & 1
\end{array}\right], \\
A=\left[\begin{array}{cccccc}
-3 & 0 & 2 & 1 & 0 & 0 \\
2 & -5 & 0 & 0 & 2 & 1 \\
0 & 3 & -4 & 0 & 0 & 1 \\
1 & 1 & 0 & -5 & 2 & 1 \\
0 & 0 & 1 & 3 & -4 & 0 \\
0 & 1 & 1 & 1 & 0 & -3
\end{array}\right], \\
B= \\
{\left[\begin{array}{cccccc}
-3 & 2 & 0 & 1 & 0 & 0 \\
0 & -3 & 0 & 0 & 1 & 2 \\
0 & 1 & -3 & 2 & 0 & 0 \\
1 & 0 & 2 & -4 & 0 & 1 \\
0 & 0 & 1 & 1 & -2 & 0 \\
2 & 0 & 0 & 0 & 1 & -3
\end{array}\right] .}
\end{gathered}
$$

Nonlinearly parameterized function satisfies

$$
\begin{aligned}
& \left\|\exp \left(-\varphi_{i}(t)\left(\begin{array}{c}
x_{j 1}^{2}(t) \\
x_{j 2}^{2}(t) \\
x_{j 3}^{2}(t)
\end{array}\right)\right)-\exp \left(-\varphi_{i}(t)\left(\begin{array}{c}
s^{2}(t) \\
s^{2}(t) \\
s^{2}(t)
\end{array}\right)\right)\right\|^{2} \\
& \leq\left\|e_{j}(t)\right\|^{2} 2 \varphi_{i}(t) \exp (-1),
\end{aligned}
$$



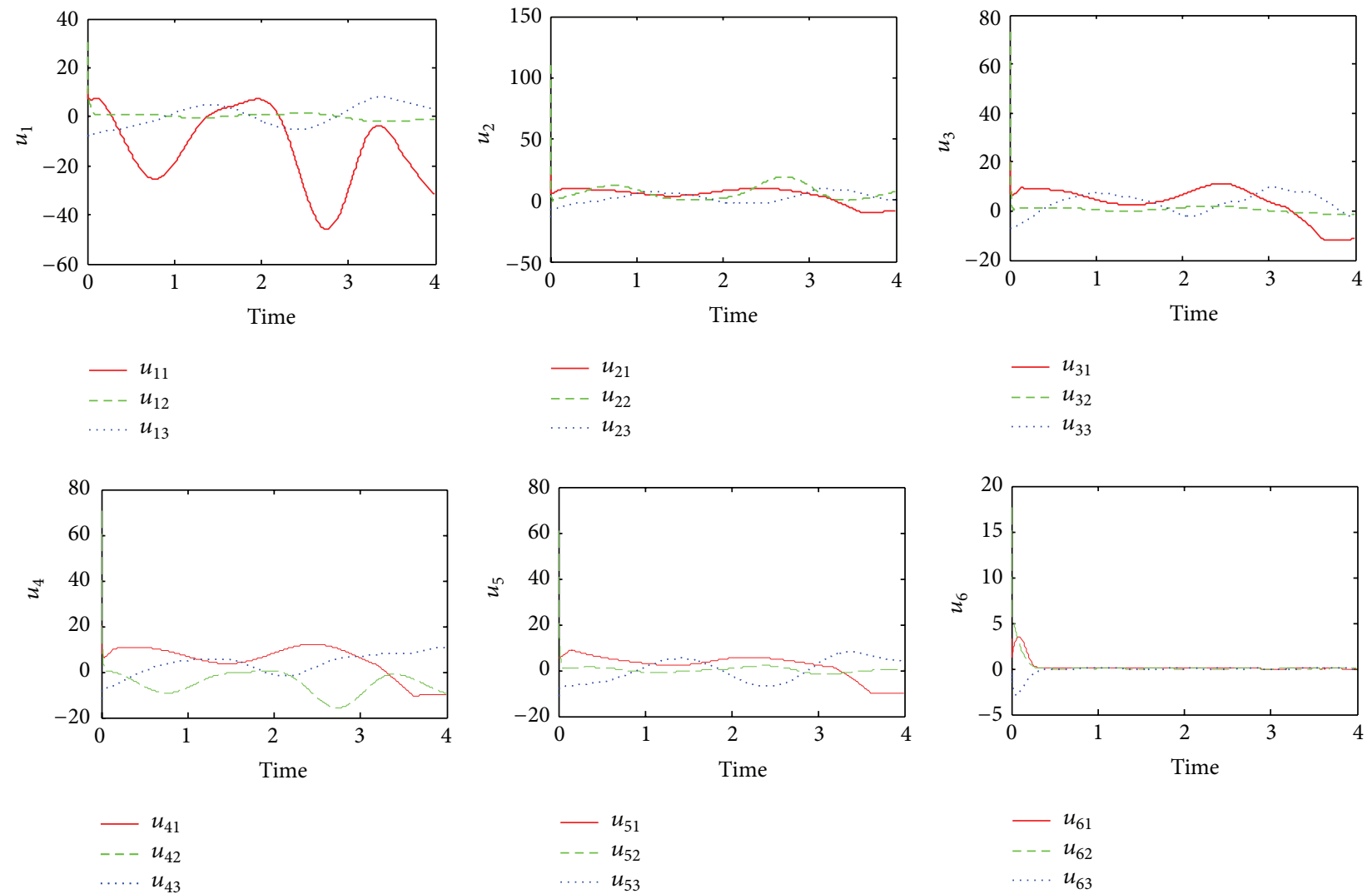

FIGURE 2: The curve of control $u_{i}(t)$ with $i=1, \ldots, 6$.

$$
\begin{aligned}
& \mid\left(\exp \left(\begin{array}{l}
\sin (2 \pi t) \\
\sin (2 \pi t) \\
\sin (2 \pi t)
\end{array}\right)-\left(\begin{array}{l}
0.1 \cos \left(\dot{x}_{j 1}(t)\right) \sin (2 \pi t) \\
0.1 \cos \left(\dot{x}_{j 2}(t)\right) \sin (2 \pi t) \\
0.1 \cos \left(\dot{x}_{j 3}(t)\right) \sin (2 \pi t)
\end{array}\right)\right) \\
& \quad-\left(\exp \left(\begin{array}{l}
\sin (2 \pi t) \\
\sin (2 \pi t) \\
\sin (2 \pi t)
\end{array}\right)+\left(\begin{array}{l}
0.1 \cos \left(\dot{s}_{j 1}(t)\right) \sin (2 \pi t) \\
0.1 \cos \left(\dot{s}_{j 2}(t)\right) \sin (2 \pi t) \\
0.1 \cos \left(\dot{s}_{j 3}(t)\right) \sin (2 \pi t)
\end{array}\right)\right) \mid \\
& \leq\left|\dot{x}_{j}(t)-\dot{s}_{j}(t)\right| .
\end{aligned}
$$

We choose $\dot{s}(t)=f_{6}(s(t))$, and the parameters are selected as follows:

$$
\begin{array}{cl}
N=6, \quad T=0.5, & \gamma=1, \quad H=5, \\
\phi_{1}(t)=0.2 \sin 8 \pi t+2, & \phi_{2}(t)=2 \cos 4 \pi t+2, \\
\phi_{3}(t)=-\sin 8 \pi t+2, & \phi_{4}(t)=\cos 8 \pi t+2, \\
\phi_{5}(t)=-2 \sin 8 \pi t+2, & \phi_{6}(t)=2 \sin 4 \pi t+2, \\
\theta=(1,2,3,1.1,1.5,1.3)^{T} .
\end{array}
$$

In the following simulations, we choose

$$
\begin{array}{cl}
q_{1}=1, \quad q_{2}=3, \quad q_{3}=2, \quad q_{4}=5, \\
q_{5}=1, \quad q_{6}=2, \quad q_{10}(t)=2 t q_{1}, \quad q_{20}(t)=2 t q_{2}, \\
q_{30}(t)=2 t q_{3}, \quad q_{40}(t)=2 t q_{4}, \\
q_{50}(t)=2 t q_{5}, \quad q_{60}(t)=2 t q_{6} .
\end{array}
$$

The initially estimated values of the unknown parameters are

$$
\widehat{\phi}(0)=\left(\begin{array}{llllll}
1 & 1 & 1 & 1 & 1 & 1
\end{array}\right)^{T}, \quad \widehat{\theta}(0)=(1,2,3,1.1,1.5,0)^{T},
$$

and the initial states are chosen as

$$
\begin{gathered}
x_{1}=\left[\begin{array}{lll}
0.01 & 0.03 & 0.02
\end{array}\right]^{T}, \quad x_{2}=\left[\begin{array}{lll}
0.03 & 0.02 & 0.03
\end{array}\right]^{T}, \\
x_{3}=\left[\begin{array}{lll}
0.04 & 0.02 & 0
\end{array}\right]^{T}, \quad x_{4}=\left[\begin{array}{lll}
0 & 0.05 & 0.03
\end{array}\right]^{T}, \\
x_{5}=\left[\begin{array}{lll}
0.1 & 0 & 0.03
\end{array}\right]^{T}, \quad x_{6}=\left[\begin{array}{lll}
0.1 & 0.01 & 0
\end{array}\right]^{T}, \\
s=\left[\begin{array}{lll}
0.1 & 0.5 & 0
\end{array}\right]^{T} .
\end{gathered}
$$

According to Theorem 14, the synchronization of the complex dynamical network can be guaranteed by the distributed adaptive controllers in (15) and the distributed adaptive learning laws when (16)-(18). Figure 1 shows the error evolutions under the designed controller. In this example, $k(\cdot, \cdot)$ is bounded function, we clearly see that the states of the network asymptotically synchronizes with the states of the desired orbit. Figure 2 depicts the time evolution of the controller, and Figure 3 shows the evolution of the estimated time-varying parameters. Figures 2 and 3 show that all signals in the network are bounded. Figures 4 and 5 show that the time-varying parameters are periodic and bounded. 


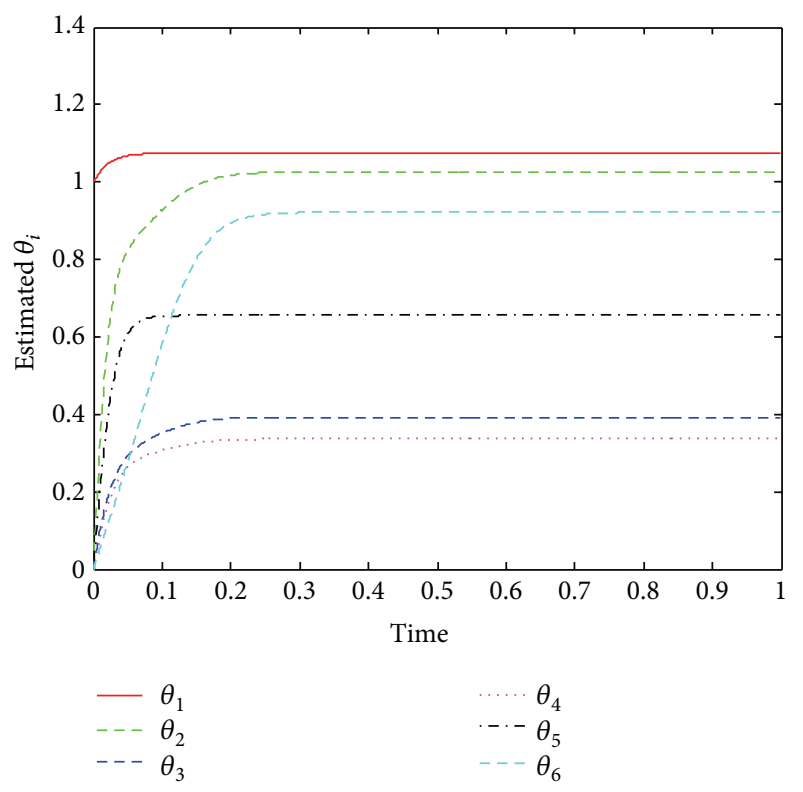

Figure 3: The curve of control $\theta_{i}$ with $i=1, \ldots, 6$.

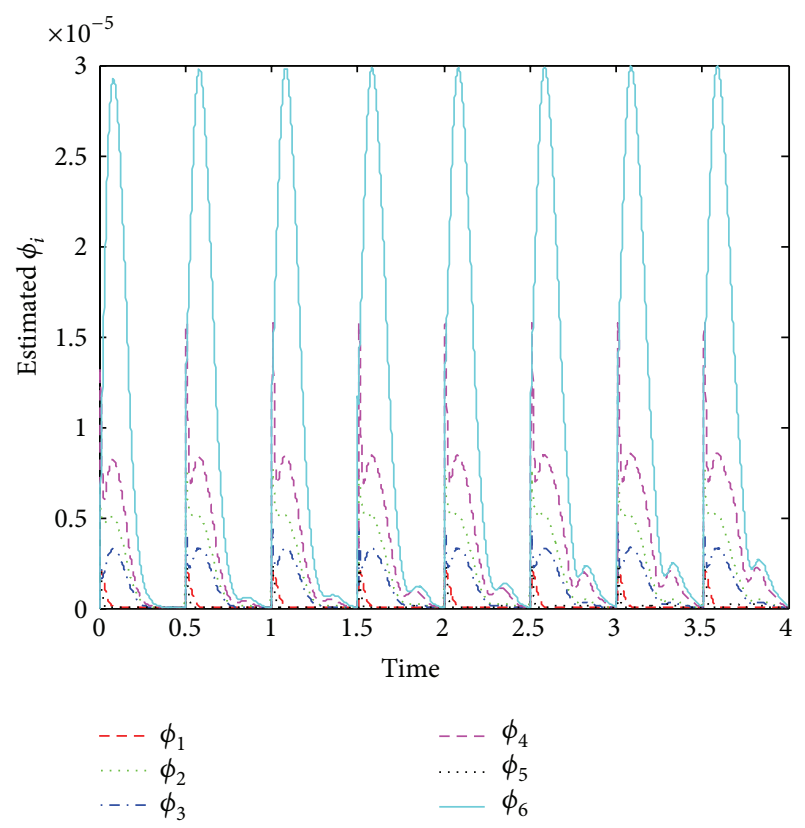

FIGURE 4: The evaluations of parameters $\phi_{i}$ with $i=1, \ldots, 6$.

Remark 15. It is not difficult to draw the evolution of other elements in parameter $M_{i}(t)$. Here, we only take the first row of $M_{11}(t)$ for example. Compared with existing results $[26,28]$, the biggest innovation of this paper is the asymptotical synchronization ability for the nonlinear neutral-type coupling complex networks under the designed controller.

\section{Conclusion}

In this paper, the synchronization problem for a complex dynamical network with nonlinearly derivative couplings is

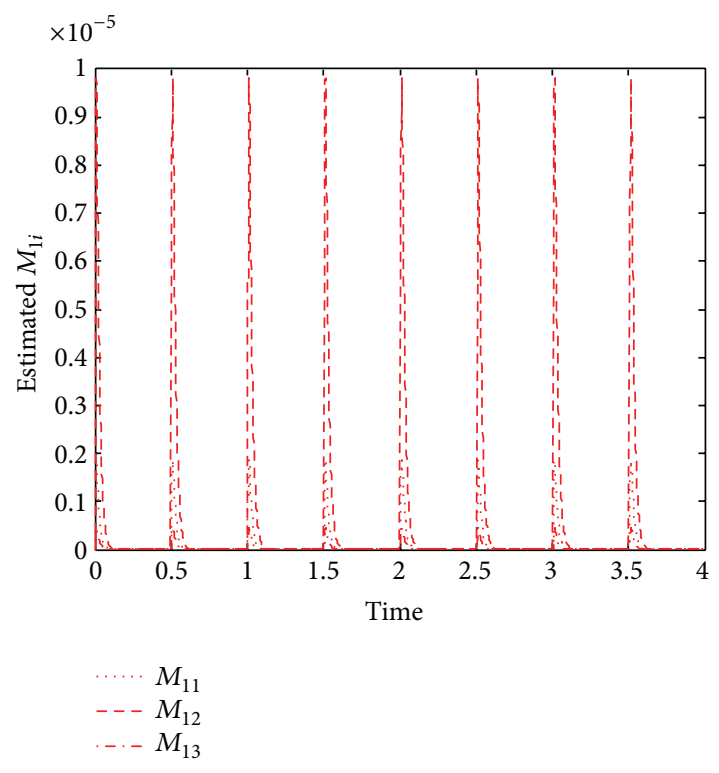

FIgURE 5: The evaluations of parameters $M_{1 i}(t)$.

solved via distributed adaptive control method. The adaptive strategies are concerned with the networks topology. By combining inequality techniques and the parameter separation, introducing the composite energy function, the convergence of the tracking error and the boundedness of the system signals are derived. Moreover, the coupling matrix is not assumed to be symmetric or irreducible. Finally, a typical example was simulated to verify the proposed theoretical results.

\section{Conflict of Interests}

The authors declare that there is no conflict of interests regarding the publication of this paper.

\section{References}

[1] J. F. Donges, Y. Zou, N. Marwan, and J. Kurths, “The backbone of the climate network," EPL, vol. 87, no. 4, Article ID 48007, 2009.

[2] R. Pastor-Satorras, E. Smith, and R. V. Solé, "Evolving protein interaction networks through gene duplication," Journal of Theoretical Biology, vol. 222, no. 2, pp. 199-210, 2003.

[3] M. Chavez, M. Valencia, V. Navarro, V. Latora, and J. Martinerie, "Functional modularity of background activities in normal and epileptic brain networks," Physical Review Letters, vol. 104, no. 11, Article ID 118701, 2010.

[4] Z. W. Gao, D. X. Kong, and C. H. Gao, "Modeling and control of complex dynamic systems: applied mathematical aspects," Journal of Applied Mathematics, vol. 2012, Article ID 869792, 5 pages, 2012.

[5] G. Chen, X. Wang, X. Li, and J. Lü, "Some recent advances in complex networks synchronization," Studies in Computational Intelligence, vol. 254, pp. 3-16, 2009.

[6] B. Song, J. H. Park, Z. G. Wu, and Y. Zhang, "Global synchronization of stochastic delayed complex networks," Nonlinear Dynamics, vol. 70, no. 4, pp. 2389-2399, 2012. 
[7] Z. G. Wu, J. H. Park, H. Su, B. Song, and J. Chu, "Exponential synchronization for complex dynamical networks with sampled-data," Journal of the Franklin Institute, vol. 349, no. 9, pp. 2735-2749, 2012.

[8] K. Yuan, J. D. Cao, and S. M. Fei, "Synchronization of coupled networks with mixed delays by intermittent control," Journal of Applied Mathematics, vol. 2012, Article ID 927609, 13 pages, 2012.

[9] T. H. Lee, J. H. Park, D. H. Ji, O. M. Kwon, and S. M. Lee, "Guaranteed cost synchronization of a complex dynamical network via dynamic feedback control," Applied Mathematics and Computation, vol. 218, no. 11, pp. 6469-6481, 2012.

[10] T. Chen, X. Liu, and W. Lu, "Pinning complex networks by a single controller," IEEE Transactions on Circuits and Systems I, vol. 54, no. 6, pp. 1317-1326, 2007.

[11] J. Zhou, J. A. Lu, and J. Lü, "Pinning adaptive synchronization of a general complex dynamical network," Automatica, vol. 44, no. 4, pp. 996-1003, 2008.

[12] M. Porfiri and M. di Bernardo, "Criteria for global pinning-controllability of complex networks," Automatica, vol. 44, no. 12, pp. 3100-3106, 2008.

[13] D. H. Ji, S. C. Jeong, J. H. Park, S. M. Lee, and S. C. Won, "Adaptive lag synchronization for uncertain complex dynamical network with delayed coupling," Applied Mathematics and Computation, vol. 218, no. 9, pp. 4872-4880, 2012.

[14] X. Y. Guo and J. M. Li, "Stochastic synchronization for timevarying complex dynamical networks," Chinese Physics B, vol. 21, no. 2, Article ID 020501, 2012.

[15] Z. G. Wu, J. H. Park, H. Su, and J. Chu, "Non-fragile synchronisation control for complex networks with missing data," International Journal of Control, vol. 86, no. 3, pp. 555-566, 2013.

[16] T. H. Lee, J. H. Park, H. Y. Jung, S. M. Lee, and O. M. Kwon, "Synchronization of a delayed complex dynamical network with free coupling matrix," Nonlinear Dynamics, vol. 69, no. 3, pp. 1081-1090, 2012.

[17] Z. W. Liu, Z. H. Guan, Y. W. Wang, and R. Q. Liao, "Synchronization of complex dynamical networks via distributed impulsive control," in Proceedings of the 48th IEEE Conference on Decision and Control held jointly with 2009 28th Chinese Control Conference (CDC/CCC '09), pp. 1714-1719, Shanghai, China, December 2009.

[18] W. Yu, P. DeLellis, G. Chen, M. di Bernardo, and J. Kurths, "Distributed adaptive control of synchronization in complex networks," IEEE Transactions on Automatic Control, vol. 57, no. 8, pp. 2153-2158, 2012.

[19] J. Xiang and G. Chen, "On the V-stability of complex dynamical networks," Automatica, vol. 43, no. 6, pp. 1049-1057, 2007.

[20] Q. Song, J. Cao, and F. Liu, "Synchronization of complex dynamical networks with nonidentical nodes," Physics Letters A, vol. 374, no. 4, pp. 544-551, 2010.

[21] J. Zhao, D. J. Hill, and T. Liu, "Global bounded synchronization of general dynamical networks with nonidentical nodes," IEEE Transactions on Automatic Control, vol. 57, no. 10, pp. 26562662, 2012.

[22] Y. Wang and J. Cao, "Cluster synchronization in nonlinearly coupled delayed networks of non-identical dynamic systems," Nonlinear Analysis, vol. 14, no. 1, pp. 842-851, 2013.

[23] J. X. Xu and Y. Tan, "A composite energy function-based learning control approach for nonlinear systems with time-varying parametric uncertainties," IEEE Transactions on Automatic Control, vol. 47, no. 11, pp. 1940-1945, 2002.
[24] J. Lü and G. Chen, "A time-varying complex dynamical network model and its controlled synchronization criteria," IEEE Transactions on Automatic Control, vol. 50, no. 6, pp. 841-846, 2005.

[25] J. Zhou, J. A. Lu, and J. Lü, "Adaptive synchronization of an uncertain complex dynamical network," IEEE Transactions on Automatic Control, vol. 51, no. 4, pp. 652-656, 2006.

[26] T. F. Wang, J. M. Li, and S. Tang, "Adaptive synchronization of nonlinearly parameterized complex dynamical networks with unknown time-varying parameters," Mathematical Problems in Engineering, vol. 2012, Article ID 592539, 16 pages, 2012.

[27] Y. Dai, Y. Cai, and X. Xu, "Synchronization criteria for complex dynamical networks with neutral-type coupling delay," Physica A, vol. 387, no. 18, pp. 4673-4682, 2008.

[28] Y. Xu, W. Zhou, J. Fang, and W. Sun, "Adaptive synchronization of the complex dynamical network with non-derivative and derivative coupling," Physics Letters A, vol. 374, no. 15-16, pp. 1673-1677, 2010.

[29] J. Wang, L. Feng, and S. K. Li, "Adaptive synchronization between two delayed complex networks with derivative coupling and non-identical nodes," in Proceedings of the International Conference on Information and Automation (ICIA '11), pp. 135140, June 2011.

[30] L. P. Deng, Z. Y. Wu, and Q. C. Wu, "Pinning synchronization of complex network with non-derivative and derivative coupling," Nonlinear Dynamics, vol. 73, no. 1-2, pp. 775-782, 2013.

[31] Y. K. Zhu, X. Y. Luo, and S. B. Li, "Exponential synchronization of complex dynamical systems with delay and derivative coupling via impulse," in Proceedings of the 10th World Congress on Intelligent Control and Automation, pp. 875-880, July 2012.

[32] Q. Bian and H. Yao, "Adaptive synchronization of bipartite dynamical networks with distributed delays and nonlinear derivative coupling," Communications in Nonlinear Science and Numerical Simulation, vol. 16, no. 10, pp. 4089-4098, 2011.

[33] W. Lin and C. Qian, "Adaptive control of nonlinearly parameterized systems: the smooth feedback case," IEEE Transactions on Automatic Control, vol. 47, no. 8, pp. 1249-1266, 2002.

[34] G. Tao, "A simple alternative to the Barbǎlat lemma," IEEE Transactions on Automatic Control, vol. 42, no. 5, p. 698, 1997. 


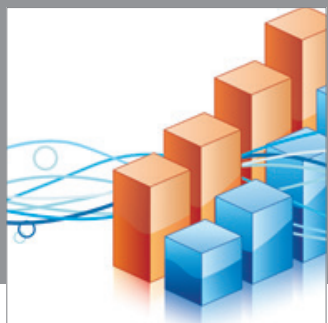

Advances in

Operations Research

mansans

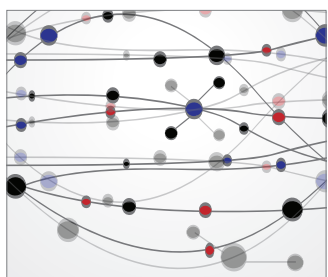

The Scientific World Journal
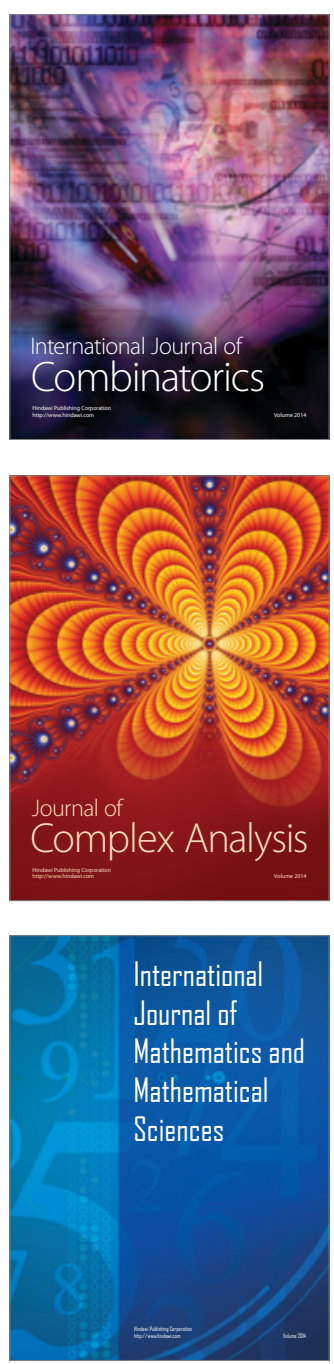
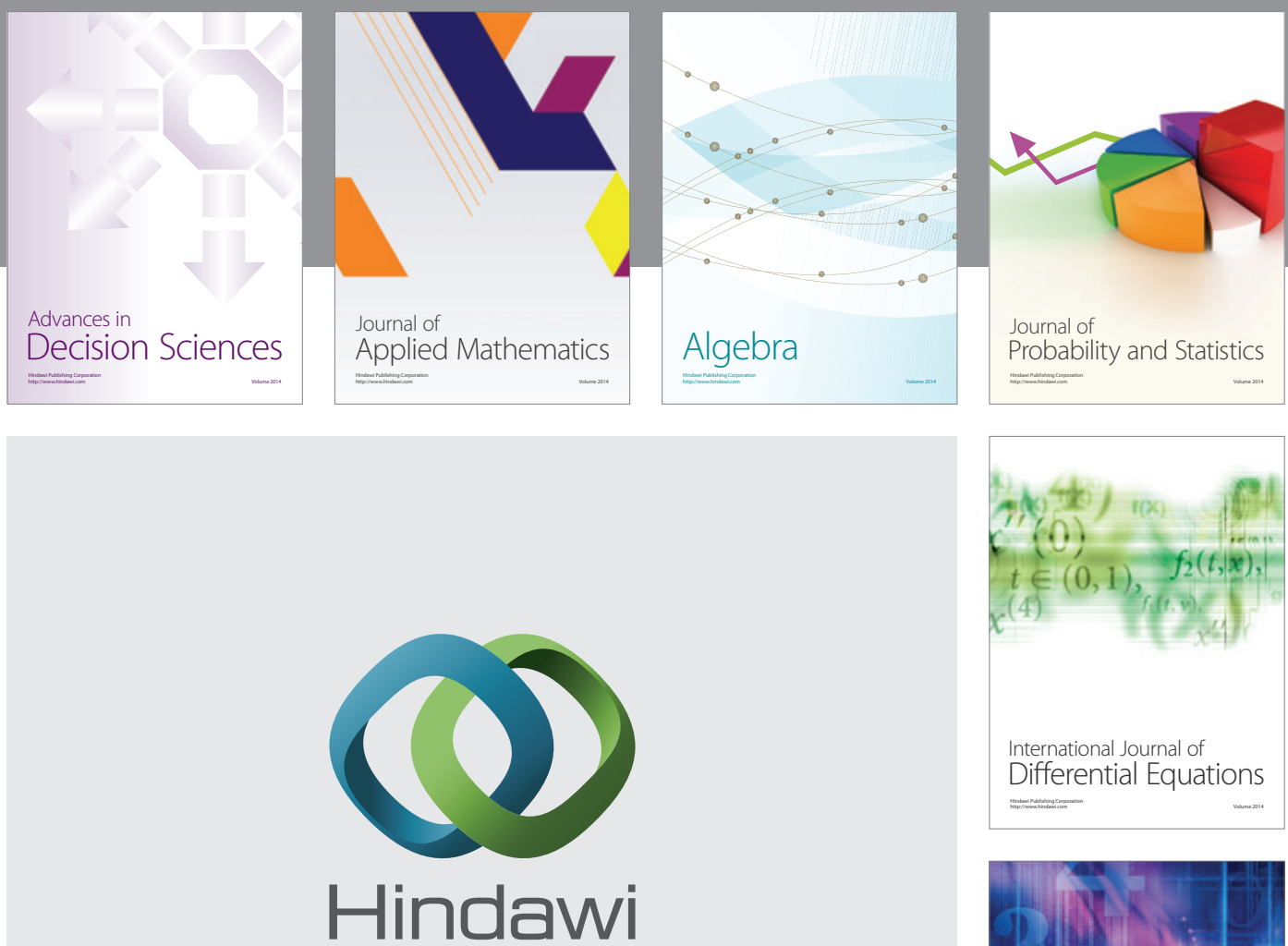

Submit your manuscripts at http://www.hindawi.com
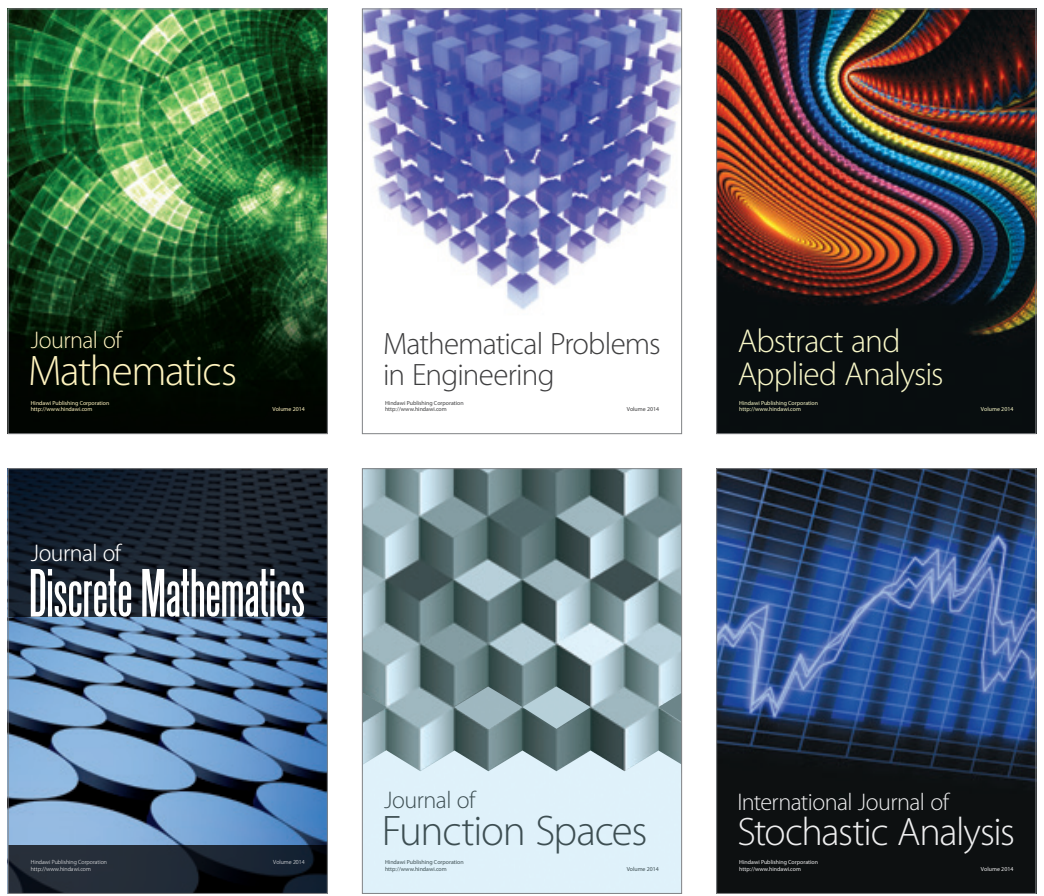

Journal of

Function Spaces

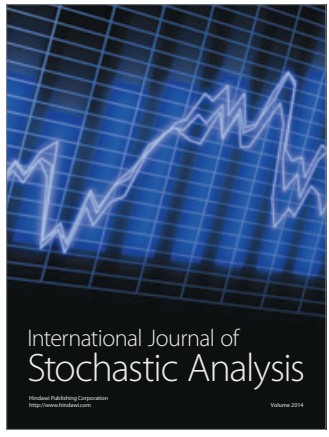

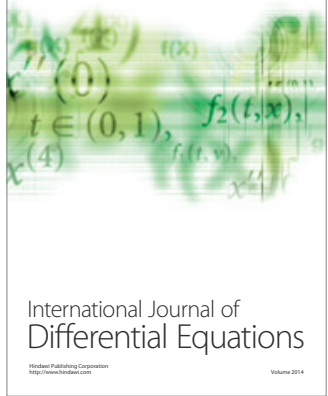
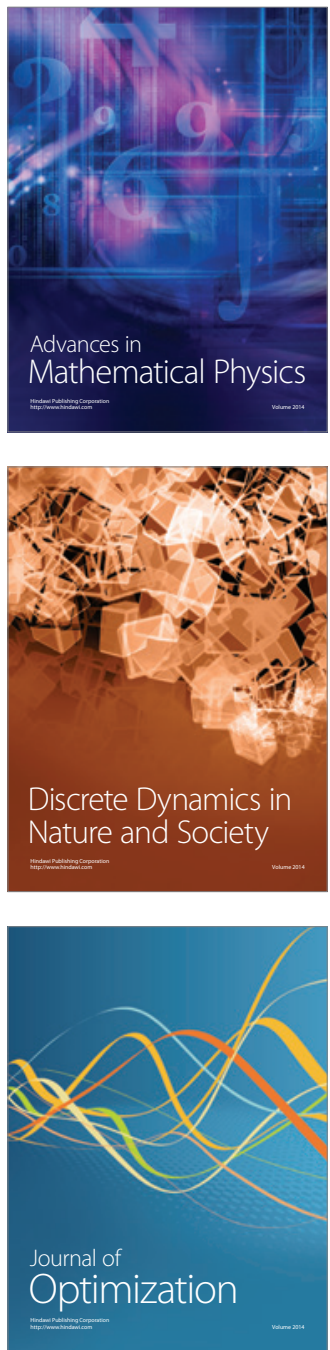